\title{
"Tree in Bud" in the Chest CT of a Patient with Granulomatosis Polyangiitis
}

\author{
Linyi Peng, Jiuliang Zhao, Qian Wang* \\ Department of Rheumatology, Peking Union Medical College Hospital (PUMCH), Chinese Academy of Medical Sciences, National \\ Clinical Research Center for Dermatologic and Immunologic Diseases (NCRC-DID), Key Laboratory of Rheumatology and Clinical \\ Immunology, Ministry of Education, Chinese Rheumatism Data Center (CRDC), Chinese SLE Treatment and Research Group \\ (CSTAR), Beijing, China
}

Received February 1, 2021; accepted February 19, 2021

\begin{abstract}
A20-year-old man was admitted because of intermittent fever, epistaxis, chest pain, and hemoptysis for 11 months. He was positive for cytoplasmic pattern of anti-neutrophil cytoplasmic antibody (ANCA) and his PR3-ANCA was $158 \mathrm{IU} / \mathrm{mL}$. The chest computerized tomography (CT) showed multiple pulmonary nodules and hollow shadows in both lungs. Fine needle aspiration lung biopsy of the pulmonary nodules revealed pathological features of granulomatous inflammation. He was diagnosed with granulomatosis polyangiitis (GPA) and was treated with high dose prednisone and oral cyclophosphamide, which resulted in a prompt response. However, he developed nocardia pneumonia and meningitis 6 months later. Cyclophosphamide was discontinued and the dose of prednisone was tapered quickly. He was treated with trimethoprim/sulfamethoxazol, combined with imipenem/
\end{abstract}

Informed consent

Informed consents have been obtained.

Conflict of Interest

None declared.

\section{References}

[1] Sacoto G, Boukhlal S, Specks U, et al. Lung Involvement in ANCA-Associated Vasculitis. Presse Med. 2020 Oct;49(3):104039. doi: 10.1016/j.lpm.2020.104039. Epub 2020 Jul 7. cilastatin. His lung and intracranial infections subsided, and the chest CT returned to clear again after the antibiotic treatment. However, the patient suffered from dry cough and fever again, and his CT scan showed a sign of "tree in bud" (Figure 1, Panel A), which was presented with multiple nodules and cavities distributed along bronchovascular pedicles. $^{[1,2]}$ After a comprehensive workup for infectious pathogens (including bacteria, mycobacteria, fungi, and nocardia), a repeated lung biopsy was conducted through video-assisted thoracic surgery (VATS). The histopathologic examination demonstrated features of chronic granuloma with necrosis, vasculitis, and microabscess, which was consistent with the diagnosis of GPA (Figure 1, Panel B). This "tree in bud" sign showed significant improvement after highdose prednisone and cyclophosphamide therapy.

[2] Szymanowska-Narloch A, Gawryluk D, Błasińska-Przerwa K, et al. Atypical Manifestations of Granulomatosis with Polyangiitis: The Diagnostic Challenge for Pulmonologists. Adv Respir Med. 2019;87(6):244-253. doi: 10.5603/ARM.2019.0062. 


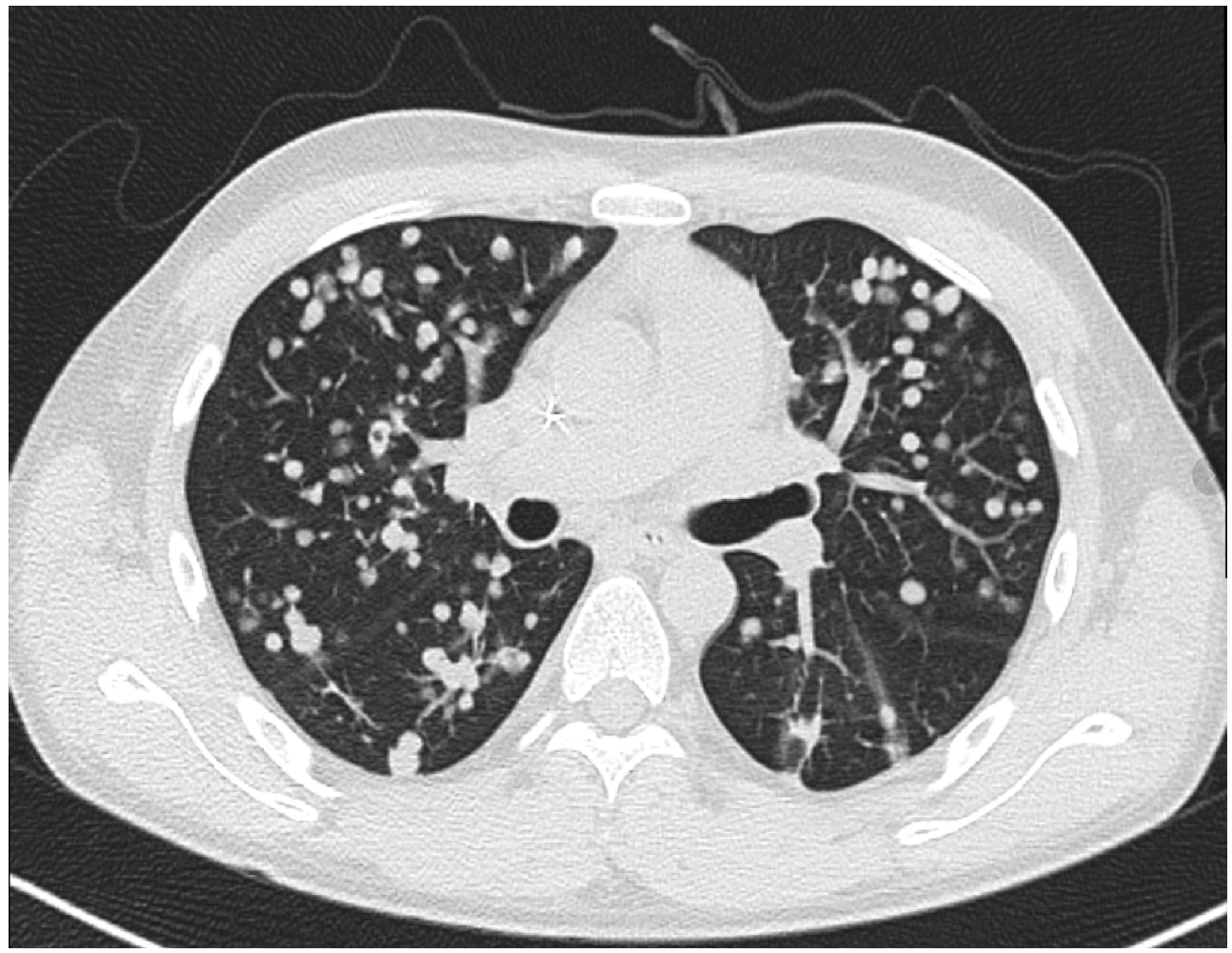

Panel A

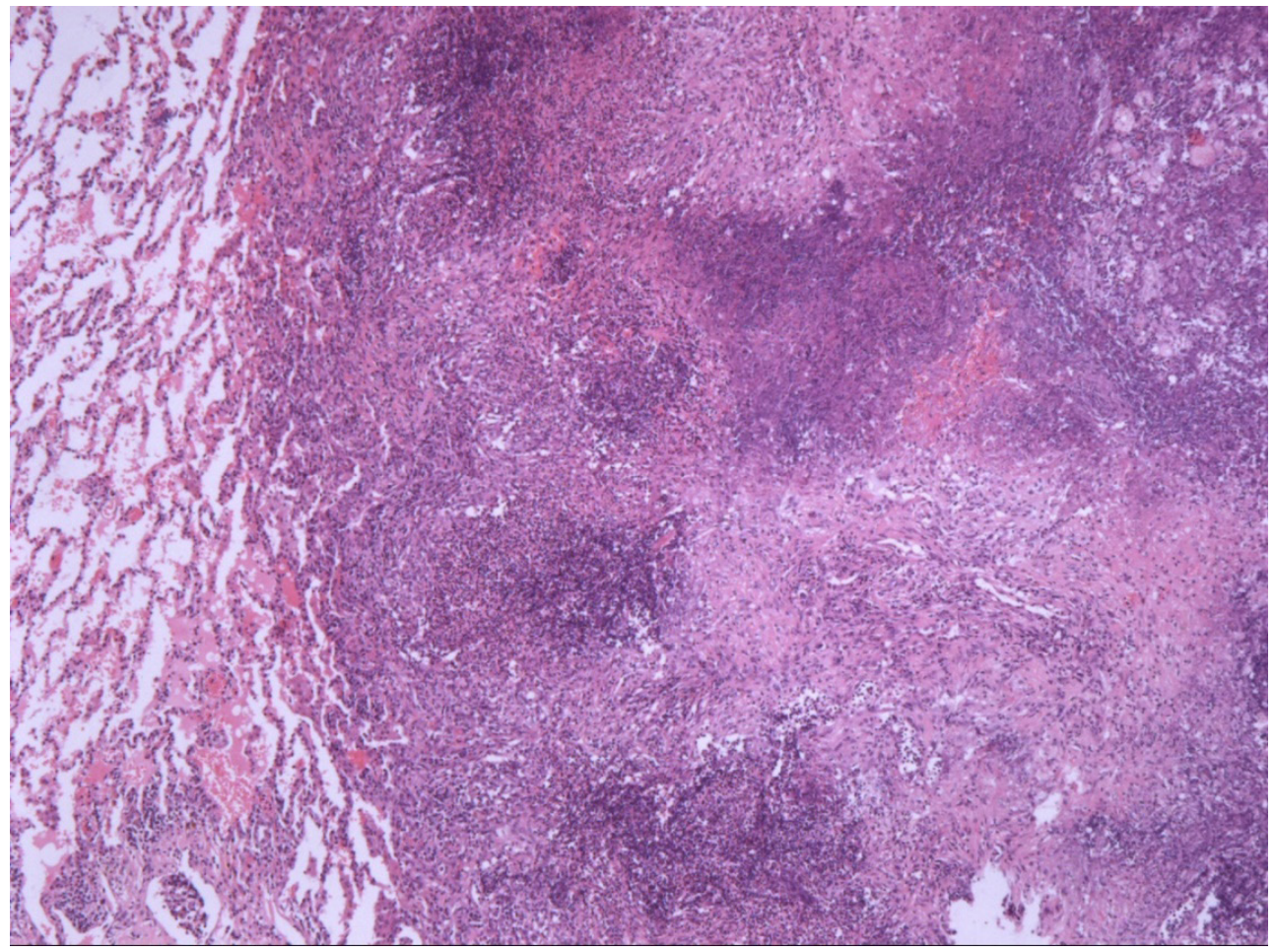

Panel B

Figure 1. The Chest tomography (CT) scan (Panel A) demonstrated a sign of "tree in bud" which was presented with multiple nodules and cavities distributed along bronchovascular pedicles. A lung biopsy was performed through video-assisted thoracic surgery (VATS), and the histopathologic examination revealed features of chronic granuloma with necrosis, vasculitis, and microabscess, which was consistent with the diagnosis of GPA (Panel B). 